\title{
First report of an isolate of Japanese iris necrotic ring virus from Australia
}

\author{
Stephen J. Wylie • Hua Li • Michael G. K. Jones
}

Received: 24 March 2012 / Accepted: 14 May 2012 / Published online: 30 May 2012

(C) Australasian Plant Pathology Society Inc. 2012

\begin{abstract}
An isolate of Japanese iris necrotic ring virus (JINRV) was detected from a plant of Iris ensata Thunb. growing in Western Australia, and the sequence of its complete genome (4019 nucleotides) was determined. The deduced replicase and coat proteins shared $75 \%$ and $72 \%$ amino acid identities, respectively, with those of the only other sequenced isolate of JINRV, from Japan. This is the first report of JINRV in Australia, and the first beyond Japan.
\end{abstract}

Keywords Flower bulb · Virus incursion .

High throughput sequencing $\cdot$ Horticulture

Japanese iris necrotic ring virus (JINRV) (family Tombusviridae, genus Carmovirus) was first described in Japan in 1982 from Japanese iris (Iris kaempferi Sieb.) (Yasukawa et al. 1982). It possessed spherical particles of about $35 \mathrm{~nm}$ in diameter with linear, monopartite, single-stranded plussense RNA. The virus was transmitted mechanically but not by aphids or through soil. It caused necrotic spindleshaped streaks or rings in the leaves of Japanese irises and latent local infection in Siberian iris (I. sanquinea Hornem. ex Donn) and rabbit-ear iris (I. laevigata Fisch.) (Yasukawa et al. 1991). The complete genome sequence of the type isolate from Japan (GenBank accession NC_002187) is 4014 nucleotides (nt) long and predicted to encode five or six overlapping open reading frames (ORF). Until now, JINRV was described only from Japan, and only one

S. J. Wylie $(\bowtie) \cdot$ H. Li $\cdot$ M. G. K. Jones

Plant Virology Group, Western Australian State Agricultural

Biotechnology Centre, School of Biological Sciences and

Biotechnology, Murdoch University,

Perth, WA 6150, Australia

e-mail: s.wylie@murdoch.edu.au sequence (Takemoto et al. 2000), that of the type isolate, was available.

Iris plants were analysed as part of a project to determine incidence and sequence diversity of potyviruses and flexiviruses in the Perth region, Western Australia. Total RNA was extracted from leaf samples of five Spanish iris (I. xiphium Desf.) and five Japanese water iris (I. ensata Thunb.) plants using the Qiagen Plant RNeasy kit. RNA samples from each species were pooled. Purification of polyadenylated RNA from total RNA using Oligo-dT labeled beads, nebulization of RNA prior to adaptor ligation, library construction, amplification, and paired-end sequencing using Illumina HiSeq 2000 technology was done by Macrogen Inc (Seoul). De novo assembly of contigs was carried out using CLC Genomics Workbench v4.8 (www.clcbio.com) and Geneious Pro v5.5.6 (Drummond et al. 2011). Parameters for the assembly of contigs were minimum overlap of $50 \%$ of read length (50 nt), $10 \%$ maximum gaps per read, $85 \%, 90 \%$ and $95 \%$ minimum overlap identity. Resulting contigs were sorted according to length. Batches of remaining contigs were subjected to Blastn, Blastx and Blastp analysis against appropriate GenBank databases. The JINRV sequences identified were edited manually to remove gaps and aberrant reads. The final virus consensus sequence was constructed using contigs from both assemblers. ORFs and identities of mature peptides, and domains encoded by them were predicted by homology after alignment with available carmovirus genomes. Pairwise identities were calculated after alignment of nt and amino acid (aa) sequences in ClustalW (Thompson et al. 1994). The phylogenetic tree of amino acid sequences was constructed using Neighbor-joining (NJ), Maximum Likelihood (ML), and Maximum Parsimony (MP) methods in MEGA5 (Tamura et al. 2011) after pairwise alignment of sequences with a Gonnet protein weight matrix, a gap open 


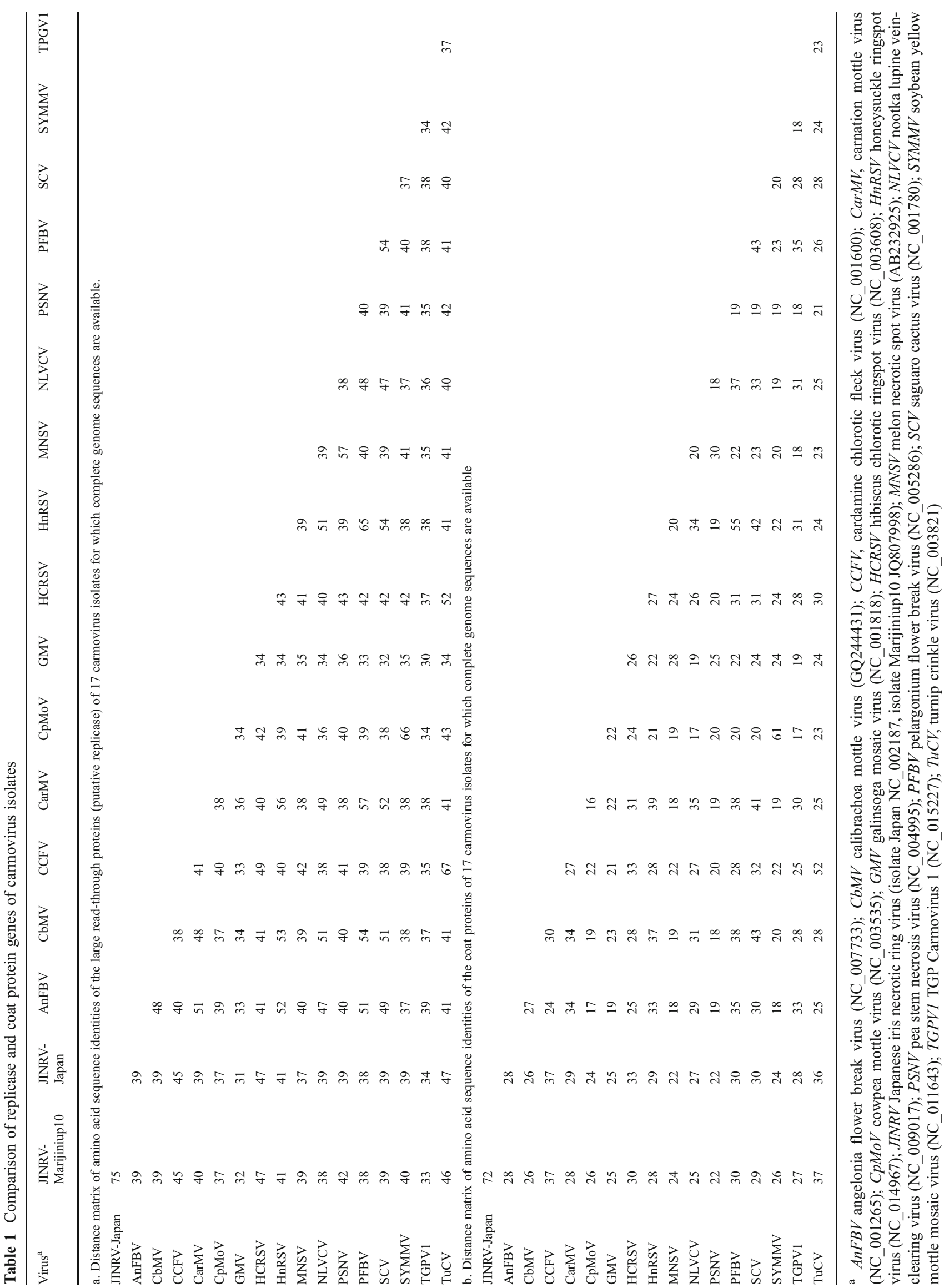


penalty of 10 , a gap separation distance of 4 , and a gap extension penalty of 0.2 . The test of phylogeny was done using 1,000 bootstrap replications.

The genome sequence of the isolate of JINRV was $4019 \mathrm{nt}$ in length, excluding the polyA tail. It was predicted to encode five ORFs. ORF1 and ORF2 encode subunits of the replicase. ORF1 encodes a protein of $27 \mathrm{kDa}$ that terminates with an amber stop codon. Read-through of this amber codon results in the synthesis of ORF2, in this case a protein of $85 \mathrm{kDa}$. The third and forth overlapping ORFs encode proteins of $8 \mathrm{kDa}$ and $12 \mathrm{kDa}$, respectively. Both proteins are thought to be involved in cell-to-cell movement (MP) of the virus. The overlapping 3'-proximal ORF5 encodes a $37 \mathrm{kDa}$ capsid protein $(\mathrm{CP})$. The isolate was named Marijiniup10 after the suburb in which it was found, and its genome sequence was granted GenBank accession JQ807998.

When the genome sequence of JINRV-Marijiniup10 was aligned with those of other carmoviruses for which a complete genome was available, it shared greatest nt identity (69 \%) with the type isolate of JINRV from Japan. It shared lower nt identities (53-57 \%) with the genome sequences of other carmoviruses. The deduced aa sequence of the large read-through protein subunit of the replicase shared $75 \%$ pairwise identity with that of JINRV-Japan and only 32 $47 \%$ identity with other carmoviruses (Table 1a). Similarly, the CP sequence had greatest aa identity $(72 \%)$ with JINRV-Japan, and shared much lower identities (22-37\%) with other carmoviruses (Table 1b). Although aa identity between the two JINRV isolates is low, the sequence identity criteria determined by the International Committee on the Taxonomy of Viruses for species demarcation for the replicase and coat proteins are $<57 \%$ and $<52 \%$ respectively (King et al. 2012). Therefore, the sequence identities between replicase and CP sequences of isolate Marijiniup10 and JINRV-Japan, and shared features of their genomic architecture, confirm them as members of the same taxon. When the replicase sequences (aa) of carmoviruses were aligned to deduce their phylogeny using NJ, ML, and MP methods, essentially the same evolutionary relationships between them were revealed with each algorithm. The NJ tree is shown (Fig. 1).

All the iris plants tested were screened individually using primers JINRVF1 (5'-CAGGGG CGCTCAGGCGACTA-3') and JINRVR1 (5'-CAACCCCCGACGCTGCCAAT-3') in an RT-PCR assay as described previously (Wylie et al. 2012). Primer JINRVF1 annealed to the 3' end of the MP and primer JINRVR 1 annealed to the $5^{\prime}$ end of the $\mathrm{CP}$ to yield a product of $376 \mathrm{bp}$. The product was sequenced directly by the Sanger method to confirm that it was derived from JINRV. The virus was detected in a single I. ensata plant. As others have noted

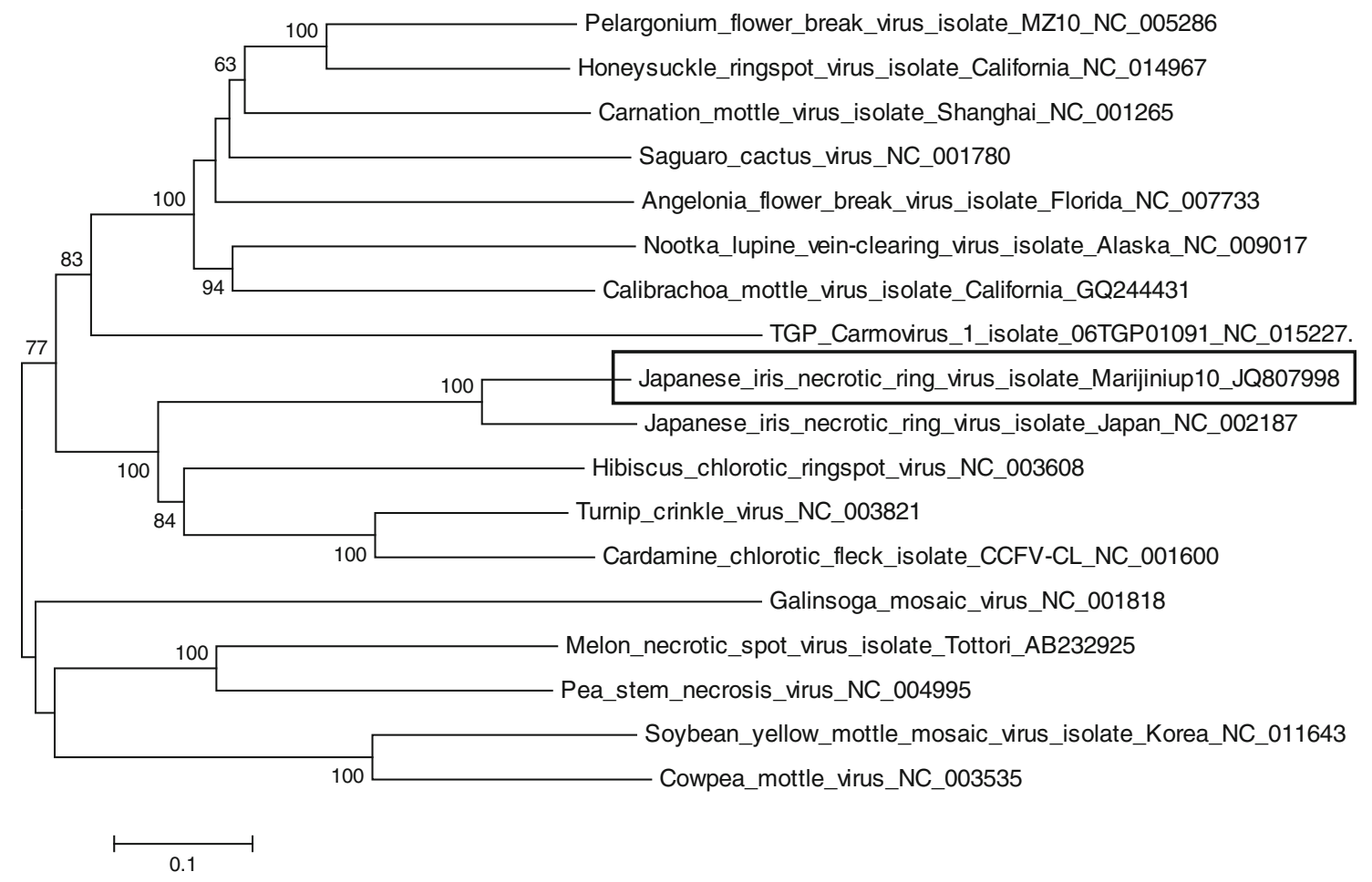

Fig. 1 Neighbor-joining tree of amino acid sequences of the large read-through protein (putative replicase) of an isolate of each species of carmovirus for which this sequence was available. Isolate names (where available) and GenBank accessions are shown. JINRV isolate
Marijiniup10 is boxed. 1,000 bootstrap replicates were done and values $>60 \%$ are shown. The tree was drawn to scale, with evolutionary distance used to infer the branch length in nucleotide substitutions per site 
for other iris host species (Yasukawa et al. 1991), the virus did not induce visible symptoms in I. ensata typical of virus infection.

Of interest is that the reads that mapped perfectly to the $3^{\prime}$ terminal 30-80 nucleotides of the JINRV genome had contiguous $3^{\prime}$ tracts of adenines of up to 43 nucleotides. Carmoviruses, including the type isolate of JINRV, are not reported to be polyadenylated (Sit and Lommel 2010; Takemoto et al. 2000). The existence of the poly (A) region cannot be explained as a cDNA synthesis or library preparation artifact because neither process involved addition of adenines. In fact, the presence of a poly (A) is required for RNA to be sequenced using this method because poly $(\mathrm{T})$ beads are utilized to capture polyadenylated RNAs while ribosomal RNAs are removed prior to cDNA library construction and sequencing. A possible explanation is that a small number of copies of the genome are, in fact, polyadenylated, but until now these have not been detected using conventional cloning and sequencing techniques. As more plant viruses are sequenced using high throughput technologies, this issue may be clarified.

To our knowledge this is the first report of Japanese iris necrotic ring virus occurring outside of Japan, and within Australia. The virus has a narrow host range and was probably inadvertently introduced to Australia on imported iris propagules, and if they were grown and underwent quarantine inspection, it was passed because no obvious symptoms of infection were evident on the leaves. Plants that are vegetatively propagated, such as irises, accumulate viruses, and when they are traded internationally, viruses infecting them are spread to new locations (Wylie et al. 2010; Wylie et al. 2011; Wylie and Jones 2012; Wylie et al. 2012). The sequencing method used was designed to detect only polyadenylated RNA viruses, so additional viruses may be present in the plant tested. This study highlights the need for more stringent examination of imported plant propagation materials at pre- and post-entry quarantine to prevent new viruses entering Australia, and the requirement for Australian producers to source virus-free stock and to also have their stocks tested for viruses prior to distribution.
Acknowledgment This study was funded by Australian Research Council Linkage Grant LP110200180

\section{References}

Drummond AJ, Ashton B, Buxton S, Cheung M, Cooper A, Duran C, Field M, Heled J, Kearse M, Markowitz S, Moir R, Stones-Havas S, Sturrock S, Thierer T, Wilson A (2011) Geneious Pro v5.5.6. http://www.geneious.com

King AMQ, Lefkowitz E, Adams MJ, Carstens EB (2012) Virus taxonomy: IXth report of the International Committee on Taxonomy of Viruses. Academic, London

Sit TL, Lommel SA (2010) Tombusviridae. In: Encyclopedia of Life Sciences. John Wiley \& Sons Ltd, Chichester. DOI: 10.1002/ 9780470015902.a0000756.pub2

Takemoto Y, Kanehira T, Shinohara M, Yamashita S, Hibi T (2000) The nucleotide sequence and genome organization of Japanese iris necrotic ring virus, a new species in the genus Carmovirus. Arch Virol 145:651-657

Tamura K, Peterson D, Peterson N, Stecher G, Nei M, Kumar S (2011) MEGA5: Molecular evolutionary genetics analysis using maximum likelihood, evolutionary distance, and maximum parsimony methods. Mol Biol Evol. doi:10.1093/molbev/msr121

Thompson JD, Higgins DG, Gibson TJ (1994) CLUSTALW: improving sensitivity of progressive multiple sequence alignment through sequence weighting, position-specific gap penalties and weight matrix choice. Nuc Acid Res 22:4673-4680

Wylie SJ, Jones MGK (2012) Complete genome sequences of seven carlavirus and potyvirus isolates on Narcissus and Hippeastrum plants in Australia, and proposals to clarify their naming. Arch Virol (in press 21 Mar 2012 AVIROL-D-12-00014)

Wylie SJ, Nouri S, Coutts BA, Jones MGK (2010) Narcissus late season yellows virus and Vallota speciosa virus found infecting domestic and wild populations of Narcissus species in Australia. Arch Virol 155:1171-1174

Wylie SJ, Li H, Jones MGK (2011) Phylogenetic analysis of allexiviruses identified on garlic from Australia. APDN. doi:10.1007/ s13314-011-0038-2

Wylie SJ, Luo H, Li H, Jones MGK (2012) Multiple polyadenylated RNA viruses detected in pooled cultivated and wild plant samples. Arch Virol 157:271-284

Yasukawa K, Ohki ST, Osaki T, Inouye T (1982) Japanese iris necrotic ring disease, a newly recognized disease caused by Japanese iris necrotic ring virus. Ann Phytopathol Soc Jpn 48:113-114

Yasukawa K, Osaki T, Inouye T (1991) Necrotic Ring Disease of Japanese Iris (Iris kaempferi Sieb.), A new disease caused by Japanese iris necrotic ring virus. Bull U Osaka Pref Ser B Agric Biol 43:21-28 